\title{
Design, Synthesis, Molecular Docking and Antibacterial Screening of Some Quinolone Compounds ${ }^{+}$
}

\author{
Lucia Pintilie 1,*, Constantin Tanase 1, Elena Mihai ${ }^{1}$, Maria Maganu ${ }^{2}$ and \\ Miron Teodor Caproiu ${ }^{2}$ \\ 1 National Institute for Chemical, Pharmaceutical Research and Development (ICCF), Bucharest, \\ 112 Vitan Av., 031299 Bucharest, Romania; cvtanase@gmail.com (C.T.); anele_drag@yahoo.com (E.M.) \\ 2 Organic Chemistry Center “C.D.Nenitescu”, 202 B Splaiul Independentei, 060023 Bucharest 6, Romania; \\ mmaganu@yahoo.com (M.M.); dorucaproiu@gmail.com (M.T.C.) \\ * Correspondence: lucia.pintilie@gmail.com \\ + Presented at the 15th International Symposium "Priorities of Chemistry for a Sustainable Development" \\ PRIOCHEM, Bucharest, Romania, 30 October-1 November 2019.
}

Published: 8 October 2019

Keywords: quinolones; fluoroquinolones; drug design; molecular docking; antimicrobial activity

Drugs belonging to the quinolone compounds are characterized by a quicker biological activity and a broad antibacterial spectrum [1-4]. Molecular, topological, and conformational characteristics on 3D quinolones optimized structure have been calculated using Spartan 14 Software. Molecular docking approach using CLC Drug Discovery Workbench 2.4 software have been realised to identify and visualize the most likely interaction ligand (quinolone) with the receptor protein. The quinolone compounds have been obtained by the Gould-Jacobs method. The compounds have been characterized by physical-chemical methods and by antimicrobial activity against Gram-positive and Gram-negative microorganisms. In this study, the DFT/B3LYP/6-311G* level of basis set has been used for the computation of molecular structure, vibrational frequencies, and energies of optimized structures. The score and hydrogen bonds formed with the amino acids from group interaction atoms are used to predict the binding modes, the binding affinities, and the orientation of the docked quinolone compound in the active site of the protein-receptor (Figures 1 and 2). The protein-ligand complex has been realized based on the X-ray structure of Bacillus cereus, which was downloaded from the Protein Data Bank (PDB ID: 1VEN). In the present study, we have reported the synthesis of some quinolone compounds. The quinolones have been evaluated for their antibacterial activity against Gram-positive and Gram-negative microorganisms.

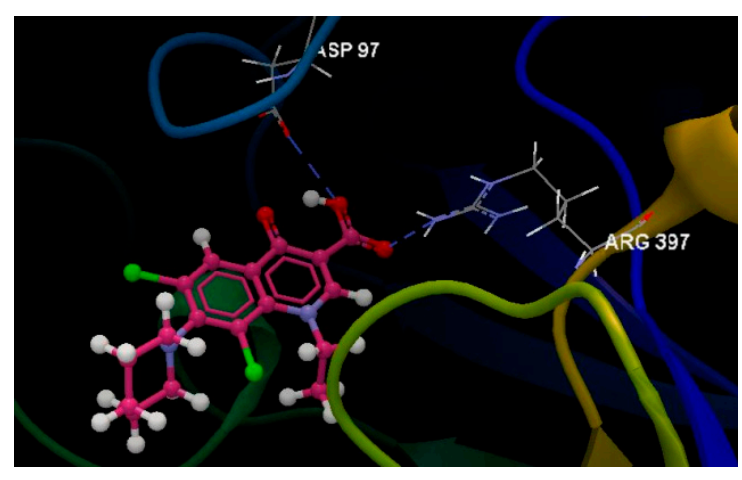

Figure 1. Docking pose of the compound 6ClPQ33. 


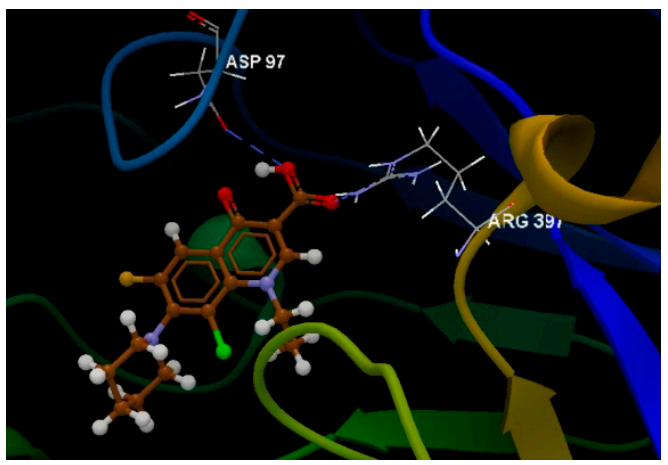

Figure 2. Docking pose of the compound FPQ33.

Structural modifications of this class of antimicrobial agents have afforded compounds with better activity aginst Bacillus cereus, Bacillus subtilis, Salmonella typhimurium, Micrococcus luteus.

Acknowledgments: This paper has been financed through the NUCLEU Program, which is implemented with the support of ANCSI, project no. PN 19-41 0102.

\section{References}

1. Cheng, G.; Hao, H.; Dai, M.; Liu, Z.; Yuan, Z. Antibacterial action of quinolones: From target to network. Eur. J. Med. Chem. 2013, 66, 555-562.

2. Gao, F.; Wang, P.; Yang, H.; Miao, Q.; Ma, L.; Lu, G. Recent developments of quinolone-based derivatives and their activities against Escherichia coli. Eur. J. Med. Chem. 2018, 157, 1223-1248.

3. Khan, T.; Sancheb, K.; Suvarna, V.; Sherje, A.; Patel, K.; Dravyakar, B. DNA gyrase inhibitors: Progress and synthesis of potent compounds as antibacterial agents. Biomed. Pharmacother. 2018, 103, 923-938.

4. Ezelarab, H.A.A.; Abbas, S.H.; Hassan, H.A.; Abuo-Rahma, G.E.D.A. Recent updates of fluoroquinolones as antibacterial agents. Arch. Pharm. Chem. Life Sci. 2018, 351, e1800141.

(c) 2019 by the authors. Licensee MDPI, Basel, Switzerland. This article is an open access article distributed under the terms and conditions of the Creative Commons Attribution (CC BY) license (http://creativecommons.org/licenses/by/4.0/). 